\title{
First reports of serious adverse drug reactions in recent weeks
}

Table 1 contains an overview of first published case reports of serious adverse drug reactions identified in the international literature in recent weeks by the drug safety alerting service Reactions Weekly. An event is serious (US FDA MedWatch definition) when the patient outcome is death, life threatening, hospitalization, disability, congenital anomaly or requires intervention to prevent permanent impairment or damage. Reactions and the customized Reactions Pharmacovigilance Service are produced by Adis, and monitor $>4,000$ journals, as well as companion journal supplements, major scientific meetings, newsletters from national centres participating in the WHO International Drug Monitoring Programme, media releases, pharmaceutical company websites, and regulatory agency websites.

Table 1 First published reports of serious adverse drug reactions recently identified by Reactions Weekly

Drug and serious adverse reaction

Blonanserin: hyponatraemia

Dipyrone: perforated sigmoid diverticulitis

Filgrastim: chemotherapy-induced neutropenic fever, followed by unmasking of immune reconstitution inflammatory syndrome cryptococcal meningitis

Granisetron: worsening post-anoxic myoclonus

Isavuconazole: liver enzyme elevation and nausea

Lansoprazole: respiratory arrest, multiorgan failure and asystole

Lomustine: disseminated Geotrichum clavatum infection

Pregabalin: agranulocytosis

Telmisartan: enteropathy
References

Bavle A. A case report of blonanserin-induced hyponatremia. J Neuropsychiatry Clin Neurosci. 2014;26(3):E43. http://dx.doi/10.1176/appi.neuropsych.13080182

Heye P, Descloux A, Singer G, et al. Perforated sigmoid diverticulitis in the presence of toxic epidermal necrolysis. Case Rep Dermatol. 2014;6(1):49-53. http://dx.doi. org/10.1159/000360129

Bahr NC, Wallace J, Frosch AEP, et al. Unmasking cryptococcal meningitis immune reconstitution inflammatory syndrome due to granulocyte colony-stimulating factor use in a patient with a poorly differentiated germ cell neoplasm. Case Rep Oncol. 2014;7(1):1-5. http://dx.doi.org/10.1159/000357666

Budhram A, Lipson D, Nesathurai S, et al. Postanoxic myoclonus: two case presentations and review of medical management. Arch Phys Med Rehabil. 2014;95(3):588-90. http://dx.doi.org/10.1016/j.apmr.2013.09.008

Peixoto D, Gagne LS, Hammond SP, et al. Isavuconazole treatment of a patient with disseminated mucormycosis. J Clin Microbiol. 2014;52(3):1016-9. http://dx.doi.org/ 10.1128/JCM.03176-13

Candar M, Gunes H, Boz BV, et al. Asystole after the first dose of lansoprazole. Am J Emerg Med. 2014;32(10):1302e3-4. http://dx.doi/10.1016/j.ajem.2014.03.051

Picard M, Cassaing S, Letocart P, et al. Concomitant cases of disseminated Geotrichum clavatum infections in patients with acute myeloid leukemia. Leuk Lymphoma. 2014;55(5):1186-8. http://dx.doi/10.3109/10428194.2013.820290

Kino H, Suzuki H, Nakamura K, et al. Clostridium difficile infection induced by pregabalin-associated agranulocytosis. Intern Med. 2014;53(18):2149-52. http://dx. doi.org/10.2169/internalmedicine.53.2085

Cyrany J, Vasatko T, Machac J, et al. Letter: telmisartan-associated enteropathy - is there any class effect? Aliment Pharmacol Ther. 2014;40(5):569-70. http://dx.doi. org/10.1111/apt.12850 